\title{
Efecto del pH, Sólidos Solubles y Zumo Adicionado sobre el color y la Vitamina C de Zumo de Naranja Agria Cocristalizado
}

\author{
Ricardo D. Andrade ${ }^{(1) \star}$, Kevin Blanquicett ${ }^{(2)}$ y Rafael D. Rangel(2) \\ (1) Grupo de Investigación GIPPAL, Facultad de Ingeniería, Universidad de Córdoba, Km 12 Vía Cereté- \\ Ciénaga de Oro, Colombia (e-mail: rdandrade@correo.unicordoba.edu.co). \\ (2) Programa de Ingeniería de Alimentos, Universidad de Córdoba, Km 12 Vía Cereté-Ciénaga de Oro, \\ Colombia.
}

${ }^{*}$ Autor a quien debe ir dirigida la correspondencia.

Recibido Abr. 14, 2016; Aceptado Jun. 7, 2016; Versión final Jul. 28, 2016, Publicado Dic. 2016

\begin{abstract}
Resumen
El objetivo de este estudio fue evaluar el efecto de la proporción de jugo adicionado (10 y 20\%), pH (3.8 y 4.5), y sólidos solubles del zumo (50 y 60 Brix) sobre el tiempo de cocristalización, el color y contenido de vitamina $\mathrm{C}$ de los cocristales de zumo de naranja agria. El zumo de naranja agria cocristalizado fue obtenido mezclando jarabe de sacarosa sobresaturada a $118^{\circ} \mathrm{C}$ con zumo de naranja agria concentrado, con agitación constante de $600 \mathrm{rpm}$. Los cocristales húmedos fueron separados y secados a $50^{\circ} \mathrm{C}$ en una estufa al vacío por 24 horas. A los cocristales secos se les determinó color y vitamina C. Los cocristales lograron retener vitamina $C$ entre un 3.94 y $5.25 \mathrm{mg} / 100 \mathrm{~g}$ en base seca. Sin embargo, se presentó una alta pérdida de vitamina C (90.64\%) durante el proceso de cocristalización con respecto al zumo fresco.
\end{abstract}

Palabras clave: cocristales, naranja agria, jarabe de sacarosa, vitamina $C$

\section{Effect of pH, Soluble Solids and Juice Added on Color and Vitamin C Content of Co-Crystallized Bitter Orange Juice}

\begin{abstract}
The effect of the proportion of juice added (10 and $20 \%$ ), $\mathrm{pH}$ (3.8 and 4.5), and soluble solids (50 and $60^{\circ}$ Brix) on time co-crystallization, color and vitamin $\mathrm{C}$ content of co-crystals of bitter orange juice was evaluated. Bitter co-crystallized orange juice was obtained by mixing supersaturated sucrose syrup at 118 ${ }^{\circ} \mathrm{C}$ with bitter orange juice concentrate, with constant agitation of $600 \mathrm{rpm}$. The wet co-crystals were separated and dried at $50^{\circ} \mathrm{C}$ in a vacuum oven for 24 hours. Color and vitamin $\mathrm{C}$ content were determined in the dry co-crystals. Co-crystals managed to retain vitamin C between 3.94 and $5.25 \mathrm{mg} / 100 \mathrm{~g}$ dry basis. However, there was high loss of vitamin C $(90.64 \%)$ during the process of co-crystallization with respect to fresh juice.
\end{abstract}

Keywords: co-crystals, bitter orange, sucrose syrup, vitamin C 


\section{INTRODUCCIÓN}

El zumo de naranja agria se destaca nutricionalmente por su contenido de vitamina C, ácido fólico y minerales como potasio, magnesio y calcio. Contiene una cantidad apreciable de beta-carotenos conocido por su carácter antioxidante; además de los ácidos oxálico, tartárico, málico y cítrico, éste último potencia la acción de la vitamina $C$. Cabe destacar, que la vitamina $C$ se pierde durante el procesamiento y almacenamiento del zumo, ya que es la vitamina más lábil debido a efectos de oxidación, interacción con otros metales, temperatura, etc. (Mendoza et al., 2015). Con el fin de conservar y extender la vida útil, tanto de los componentes del zumo como de sus subproductos, estos se pueden almacenar en forma encapsulada (Yujing et al., 2014). Entre los métodos para encapsular, la cocristalización es usada con frecuencia en la industria alimentaria. La cocristalización es una técnica de encapsulación que utiliza generalmente sacarosa como matriz para la incorporación de un núcleo de cristalización (Varzakas y Constantina, 2015). En el proceso de co-cristalización de la sacarosa, su estructura cristalina se modifica para proporcionar una matriz porosa en la que el ingrediente activo es incorporado. El proceso de cocristalización implica la concentración de jarabes de sacarosa por evaporación a temperatura elevada hasta que se consigue la cristalización espontánea de sacarosa (López et al., 2016).

La cocristalización mejorar diversas propiedades físicas de los compuestos activos, tales como: solubilidad, estabilidad, dispersabilidad, humectabilidad, fluidez y estabilidad (Shimono et al., 2015). Diversos autores han estudiado el proceso de cocristalización en numerosos materiales activos. Por ejemplo Bhandari et al. (1998) encapsularon miel de abeja en jarabe de sacarosa, usando diferentes proporciones de azúcar:miel $(90: 10,85: 15,80: 20)$, obteniendo un material relativamente seco con buenas propiedades de flujo. Astolfi et al. (2005) estudio la cinética de cocristalización de jugo de maracuyá en sacarosa, variando el pH (3.5 y 4.5), y jugo añadido (10\% y 15\%); evidenciando que la velocidad de cristalización se acelera al aumentar el $\mathrm{pH}$ y al reducir el jugo adicionado. Por otra parte, agentes activos amargos, como sulfato de zinc, se pueden cocristalizar con azúcar para enmascarar el sabor y pueden ser comprimidos directamente para ser utilizados en la industria farmacéutica y de dulces (López-Córdoba et al., 2016). El objetivo de este estudio fue evaluar el efecto del $\mathrm{pH}$, sólidos solubles y cantidad de zumo adicionado sobre el tiempo de cocristalización, color y vitamina $\mathrm{C}$ de cocristales de zumo de naranja agria.

\section{MATERIALES Y METODOS}

Materia prima: Los frutos de naranja agria (Citrus aurantium L.) se recolectaron en la finca San Antonio seis perteneciente al corregimiento de Malagana de Ciénaga de Oro, con un estado de maduración de grado 2 de acuerdo a lo reportado por Barón y Villa (2013). Las naranjas se enjuagaron con agua a presión y desinfectaron por inmersión en cloro diluido al $0.5 \%$ durante $10 \mathrm{~min}$.

Caracterización del zumo de naranja agria: la obtención del zumo de naranja agria se realizó en un exprimidor mecánico filtrándolo con tela fina. Al zumo se le realizaron los siguientes análisis: Acidez titulable (Método AOAC 942.15.), Humedad (Método AOAC 20.013.), pH (Método AOAC 981.12.), Solidos solubles (Método AOAC 932.12.) y Vitamina C (Método AOAC 967.21.).

Cocristalización del zumo: Al zumo de naranja agria se le ajusto el pH (3.8, 4.15 y 4.5) empleando Hidróxido de Calcio. Luego el zumo se llevó a un rota-evaporador (Modelo Laborota 4000 eco, Heidolph), con el fin de concentrar el zumo hasta la concentración de solidos solubles a evaluar. El zumo concentrado fue etiquetado y almacenado en refrigeración.

Para preparar los jarabes de sacarosa se pesan $300 \mathrm{~g}$ de azúcar y $50 \mathrm{ml}$ de agua, con el fin de obtener una solución concentrada de $70^{\circ}$ Brix. Esta solución es sometida a calentamiento en una plancha eléctrica a $180^{\circ} \mathrm{C}$ con agitación magnética a $1000 \mathrm{rpm}$, hasta observar una coloración blanca, aproximadamente a una temperatura de $118^{\circ} \mathrm{C}$, la cual indicaba el grado de sobresaturación requerido para que ocurra la cristalización espontanea al enfriarse. En este punto el jarabe es retirado de la fuente de calentamiento y se le añade la cantidad estipulada de zumo concentrado. La mezcla es sometida a agitación constante de 600 rpm, usando un agitador mecánico portátil (Modelo 906, Maquita) dotado con aspas metálicas rectas de 4 $\mathrm{cm}$ de diámetro. Al observar la formación de un material sólido particulado la agitación fue detenida.

El material particulado es separado y sometido a un proceso de secado a $50^{\circ} \mathrm{C}$ en una estufa al vacío (Modelo 037, Marconi) durante 24 horas, seguido por una molturación y cribado utilizando un colador comercial. Finalmente, el producto se depositó en bolsas de polietileno transparente tipo Ziploc y almacenadas hasta su posterior análisis. Al zumo cocristalizado se le realizaron los siguientes análisis:

Tiempo de Cocristalización: se determinó de acuerdo a lo propuesto por Astolfi et al. (2005), midiendo el tiempo de agitación transcurrido entre la adición del jugo y la obtención de un material sólido granular. 
Color: la medición del color se llevó a cabo en un Colorímetro ColorFlex EZ marca HunterLab, determinando la luminosidad $L^{*} \quad\left(100=\right.$ blanco, $0=$ negro), $a^{*}$ (positivo=rojo, negativo=verde) y $b^{*}$ (positivo=azul, negativo=amarillo), con iluminante estándar D65, observador 10ํy diametro de observación de $25.4 \mathrm{~mm}$.

Vitamina $C$ : para la medición de vitamina $C$ se usó el método de la AOAC 967.21. Se utilizó para titular una solución de 2.6-diclorofenolindofenol a 400 ppm. Aproximadamente $5 \mathrm{~g}$ de cada una de las muestras, previamente diluidas en $20 \mathrm{ml}$ de ácido oxálico $2 \%$, hasta obtener un color rosa constante. Además, se tituló $0.2 \mathrm{ml}$ de una solución patrón de ácido ascórbico $0.2 \%(\mathrm{p} / \mathrm{v})$ y $0.2 \mathrm{ml}$ de agua destilada empleada como blanco. Los resultados se expresaron en mg de Vitamina C por $100 \mathrm{~g}$ de muestra.

Diseño experimental y análisis: para estudiar el efecto del pH (3.8 y 4.5), concentración de sólidos solubles en el zumo (50 y 60 Brix) y cantidad de zumo concentrado adicionado (10 y 20\%) sobre el tiempo de cocristalización, color y vitamina $\mathrm{C}$ de los cocristales de zumo de naranja agria, se empleó un diseño de Box-Behnken, con 3 puntos centrales y 1 replica. Un análisis de varianza se realizó para determinar los efectos significativos. Se aplicó un modelo polinomial de segundo orden para predecir el comportamiento experimental de las variables de repuesta. El análisis del diseño se realizó utilizando el programa estadístico JMP 9.0.1 (SAS Institute).

\section{RESULTADOS Y ANÁLISIS}

En la tabla 1 se presentan los valores fisicoquímicos determinados al zumo de naranja agria. Las muestras de zumo de naranja agria analizadas presentaron valores semejantes a lo reportado por Garau et al. (2007). En la composición de la naranja agria se destaca la vitamina C, con un contenido de $49.1 \pm 1.94 \mathrm{mg} / 100 \mathrm{~g}$. Una cantidad inferior a la naranja valenciana $(78.5 \mathrm{mg} / 100 \mathrm{~g})$ pero superior a la reportada en mandarina (32.5 mg/100 g) (Couto y Canniatti-Brazaca, 2010).

Tabla 1. Caracterización del zumo de la naranja agria

\begin{tabular}{|l|l|}
\hline Parámetro & Cantidad \\
\hline Ácido cítrico (\%) & $4.13 \pm 0.08$ \\
\hline Sólidos solubles, ${ }^{\circ}$ Brix & $9.72 \pm 0.24$ \\
\hline Humedad (\%) & $90.6 \pm 0.08$ \\
\hline $\mathrm{pH}$ & $2.94 \pm 0.05$ \\
\hline Vitamina C (mg/100g) & $49.1 \pm 1.94$ \\
\hline
\end{tabular}

Tiempo de Cocristalización: Los valores del tiempo de cocristalización estuvieron comprendido entre 830 y 2760 s. Estos valores están de acuerdo a los reportados para zumo de maracuyá cocristalizado de 750 a $2700 \mathrm{~s}$ (Montes at al., 2006). Los factores $\mathrm{pH}(\mathrm{p}=0.001)$, concentración del zumo ( $\mathrm{p}=0.001)$, porcentaje de zumo adicionado $(\mathrm{p}=0.001)$, y el factor cuadrático $\mathrm{pH}(\mathrm{p}=0.0022)$ afectaron el tiempo de cocristalización. En las figuras 1 (a) y 1 (b) se evidencia el comportamiento del tiempo de cocristalización, el cual aumenta un $95 \%$ a medida que el zumo adicionado pasa de 10 a 20\%. Esto posiblemente se deba a qué durante la adición del zumo concentrado se produce un enfriamiento de la solución de sacarosa, lo cual promueve el paso de la zona metaestable a una zona lábil. De igual forma, al adicionar zumo concentrado, se provoca una dilución de la mezcla, disminuyendo a su vez el grado de sobresaturación; siendo esta dilución mayor cuando los niveles de jugo adicionado y/o los sólidos solubles aumentan (Astolfi et al., 2005).

(a)



(b)

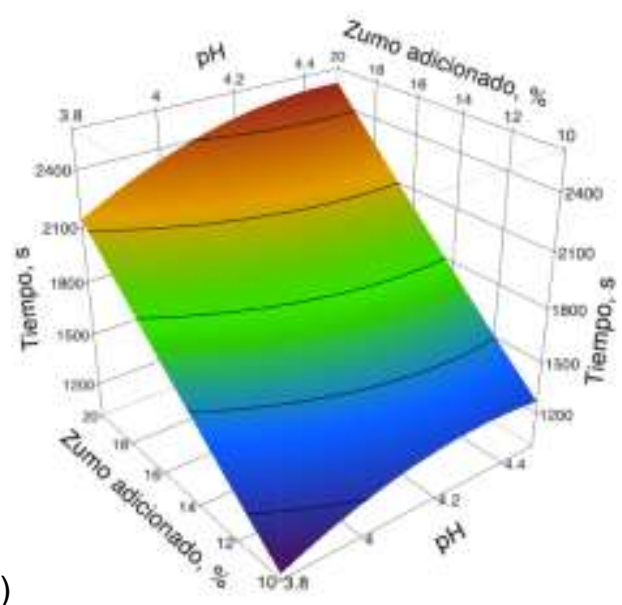

Fig.1: Gráfico de superficie de respuesta del tiempo de cocristalización en función del pH con respecto a (a) sólidos solubles, ํrix y (b) zumo adicionado. 
Color: El parámetro de color luminosidad $\left(L^{*}\right)$ mostró diferencia significativa $(p<0.05)$ con respecto al factor $\mathrm{pH}(\mathrm{p}=0.0008)$ y el factor cuadrático porcentaje de zumo adicionado $(\mathrm{p}=0.046)$. En la figura 2 se muestra que a medida que el pH aumenta (3.8 a 4.5), la luminosidad de los cocristales de zumo de naranja agria aumenta, siendo éste cambio de aproximadamente un $23 \%$. Esto posiblemente se deba a qué el Hidróxido de Calcio con el cual se ajustó el $\mathrm{pH}$, es un compuesto de color blanco que tiende a aumentar los valores de $L^{*}$ en el sistema. De acuerdo a Jacho y Vásquez (2011) la adición de Hidróxido de Calcio en el jugo de sábila y naranja tiene una relación directa sobre la luminosidad del jugo. López-Córdoba et al. (2014) reportaron que en cocristales del extracto de yerba mate se presenta una tendencia cuadrática de $L^{*}$ en función del extracto adicionado (3, 5 y 10\%), siendo estos valores de 55.3, 64.4 y 55.9, respectivamente.

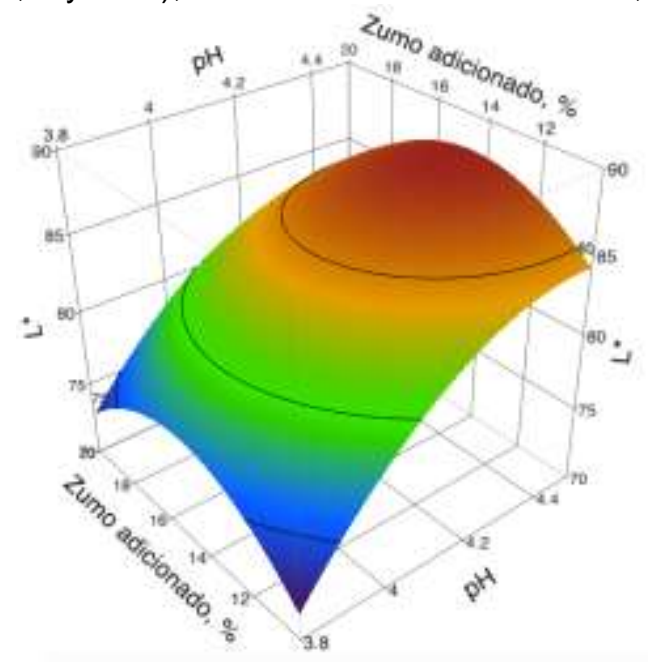

Fig. 2: Gráficos de superficie de respuesta de la luminosidad $\left(\mathrm{L}^{*}\right)$ en función del pH y el porcentaje de zumo adicionado.

La cromaticidad verde $(-)$ roja $(+)$ se vio influenciada significativamente $(p<0.05)$ con los factores: $p H$ $(p=0.0001)$ y zumo adicionado $(p=0.0203)$, los factores cuadráticos $p H(p=0.002)$, y sólidos solubles $(p=0.0056)$, y la interacción sólidos solubles - zumo adicionado ( $p=0.0015)$. En la figura $3(a)$ se observa que a medida que el $\mathrm{pH}$ aumenta la cromaticidad rojiza disminuye, siendo ésta reducción del $77.9 \%$. Esto probablemente esté relacionado con la presencia ácido ascórbico, ya que a pH más ácidos posee mayor disponibilidad para reaccionar con los componentes responsables del color dando como resultado una disminución de la cromaticidad rojiza (Martínez et al. 2011). El efecto de la interacción sólidos solubles zumo adicionado, se puede apreciar en la figura 3(b). Cuando los sólidos solubles del zumo de naranja agria son $50^{\circ}$ Brix, la cromaticidad rojiza disminuye un $72 \%$ con el aumento del zumo adicionado (10 a $20 \%$; mientras que a $60^{\circ}$ Brix, la cromaticidad rojiza aumenta, cuando aumenta el contenido de zumo adicionado (10 a 20\%), este aumento es de aproximadamente un 114\%. Manayay et al. (2013) observaron una disminución permanente del color y el croma con respecto a las variables temperatura y tiempo en la pulpa de mango (16 y $28^{\circ}$ Brix). La cromaticidad amarilla $(+)$ azul $(-)$ presentó diferencia significativa $(p<0.05)$ con respecto al factor $\mathrm{pH}(\mathrm{p}=0.0437)$, el factor cuadrático sólidos solubles $(\mathrm{p}=0.0188)$, y la interacción $\mathrm{pH}$ - zumo adicionado $(p=0.0066)$. En las figuras $4(a)$ y $4(b)$ se observa que la cromaticidad amarilla tiende hacia un espectro menos amarillo cuando el $\mathrm{pH}$ aumentan.

(a)

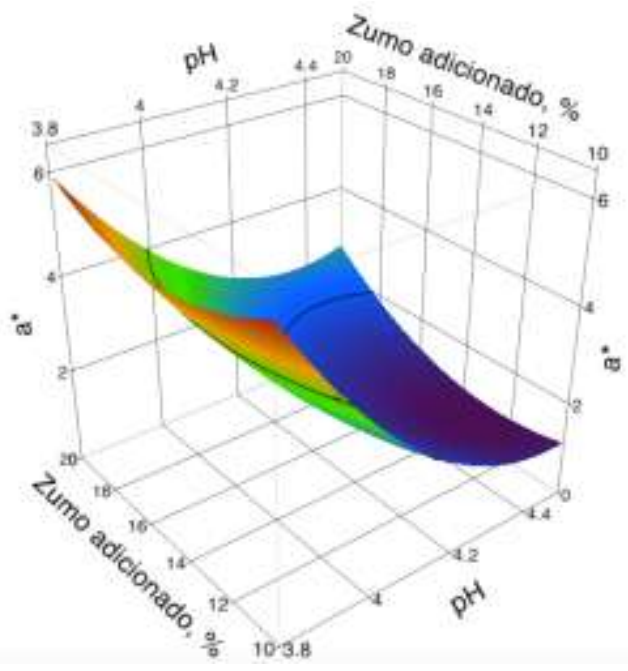

(b)

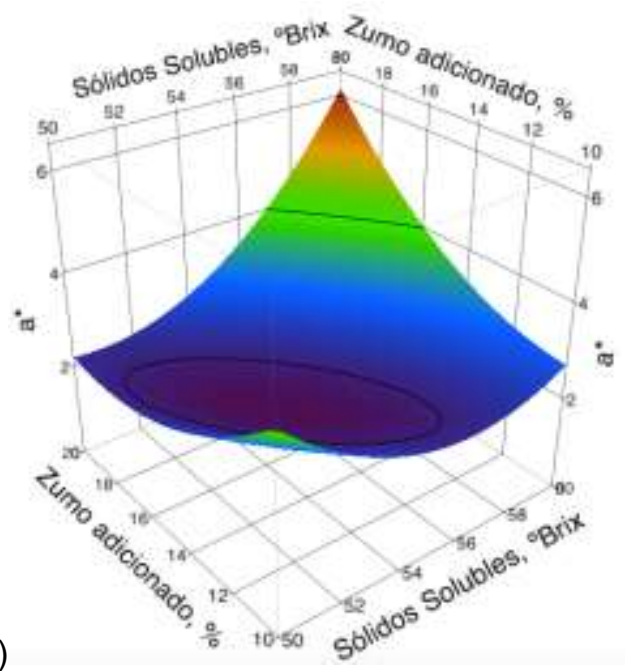

Fig. 3: Gráficos de superficie de respuesta del parámetro de color a* en función del (a) pH y solidos solubles y (b) solidos solubles y zumo adicionado. 
Torregrosa et al. (2005) al evaluar el efecto del pH sobre los carotenoides en zumo de naranja y zanahoria al ser sometido a pulsos eléctricos; encontraron que existe un aumento de las xantofilas (precursoras de la cromaticidad amarilla) tales como la 9-cis-vioxalantina, neoxan-tina, anteraxantina y la cis- $\beta$-criptoxantina, siendo éste aumento directamente proporcional a pH más ácidos.

(a)

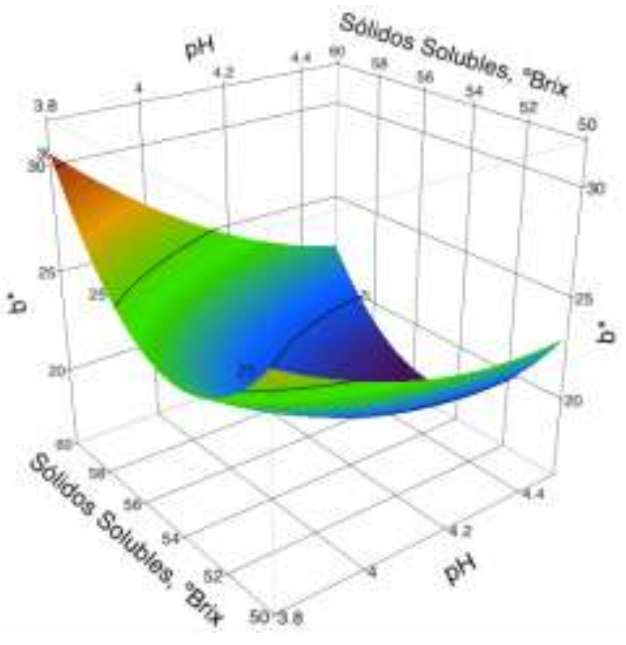

(b)

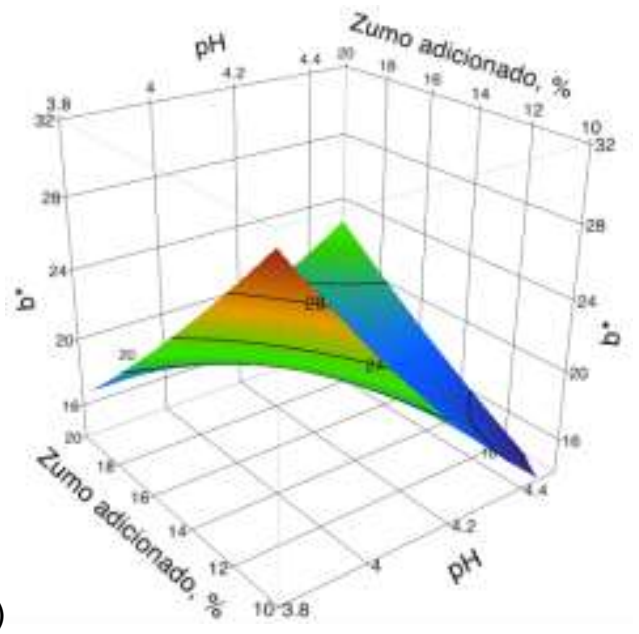

Fig. 4: Gráficos de superficie de respuesta del parámetro de color b* en función del (a) pH y solidos solubles ( ${ }^{\circ}$ Brix) y (b) $\mathrm{pH}$ y porcentaje de zumo adicionado.

Vitamina $C$. El contenido de vitamina $C$ mostró diferencia significativa $(p<0.05)$ con respecto al factor lineal $(p=0.0002)$ y cuadrático $(p=0.0028)$ del zumo adicionado, y la interacción $\mathrm{pH}$ - zumo adicionado $(p=0.046)$. Los cocristales lograron retener vitamina $C$ entre un 3.94 y $5.25 \mathrm{mg} / 100 \mathrm{~g}$ (base seca), lo cual concuerda con los datos reportados para jugos cocristalizados de limón, 4.97 - $6.52 \mathrm{mg} / 100 \mathrm{~g}$ (Astolfi, 2003) y maracuyá cocristalizado, $5.39 \mathrm{mg} / 100 \mathrm{~g}$ (Montes et al., 2006). Sin embargo, se presentó una alta pérdida de vitamina C (90.64\%) durante el proceso de cocristalización con respecto al zumo fresco, esto concuerda con lo reportado para jugo de maracuyá y limón de 85 y 89\%, respectivamente (Astolfi, 2003). Esta reducción puede estar asociada al momento que la sacarosa se incorpora en la matriz, lo cual causa un efecto de dilución de la vitamina $\mathrm{C}$. A esto se le suma el efecto combinado de la agitación, la temperatura $\left(45^{\circ} \mathrm{C}\right)$ y la exposición a la luz durante su procesamiento para su concentración. En la figura 5 se observa la tendencia de aumento del contenido de vitamina C (24.25\%) en la medida que el zumo de naranja agria adicionado pasa de $10-20 \%$, principalmente a pH altos. Este incremento puede estar asociado con el aumento de la concentración de vitamina $\mathrm{C}$ sobre la materia seca, por efecto de la adición del zumo teniendo en cuenta las proporciones propuestas. De igual forma, la cantidad de zumo adicionado probablemente minimizó el impacto combinado de la agitación y temperatura, en la degradación de la vitamina $\mathrm{C}$ durante el proceso de concentración, cocristalización y secado (Astolfi et al., 2005).



Fig. 5: Gráfico de superficie de respuesta para la vitamina $\mathrm{C}$ en función del pH y zumo adicionado

\section{CONCLUSIONES}

La cocristalización del zumo de naranja agria constituye una alternativa prometedora como fuente importante de ingredientes activos, como contenido de vitamina C. La cantidad de zumo de naranja agria adicionada y el $\mathrm{pH}$, deben ser lo mas alto posible para garantizar que los cocristales del producto tengan 
altas concentraciones Vitamina C, pero esto ocasiona altos tiempos de cocristalización y disminución de la cromaticidad rojiza y amarilla, que son características de los zumos de citricos. El contenido de sólidos solubles no afecta el contenido de Vitamina C de los cocristales de zumo de naranja agria, pero este parámetro se debe mantener en los niveles bajos que garanticen menores tiempos de cocristalización.

\section{AGRADECIMIENTOS}

Los autores agradecen a la División de Investigaciones de la Universidad de Córdoba, y en especial a la convocatoria "Proyectos de investigación en menor cuantía para financiar trabajos de grado en la modalidad de proyectos de investigación de pregrado" 2015, por el apoyo económico a esta investigación.

\section{REFERENCIAS}

Astolfi, Z. Encapsulação de sucos de frutas por co-cristalização com sacarose. Tesis de Maestría. Universidad Estatal Paulista. Júlio de Mesquita Filho. São José do Rio Preto (2003)

Astolfi, Z., A.C. Souza, E.C.D. Reipert, y V.R.N. Telis. Encapsulação de suco de maracujá por cocristalização com sacarose: cinética de cristalização e propriedades físicas. Ciência e Tecnologia de Alimentos, Campinas, 25(4), 795-801 (2005)

Baron, R. y A. Villa. Evolución de los parámetros de calidad de naranja valencia producida en el municipio de Chimichagua, Cesar - Colombia. Revista Temas Agrarios, 18(1), 66-74 (2013)

Bhandari, B., N. Datta, B. D’arcy y G. Rintoul. Co-Crystallization of honey with sucrose. LWT - Food Science and Technology, 31 (2),138-142 (1998)

Couto, M.A.L. y S.G. Canniatti-Brazaca. Quantificação de vitamina C e capacidade antioxidante de variedades cítricas. Ciência e Tecnologia de Alimentos, 30(1),15-19 (2010)

Garau, M.C., S. Simal, C. Rosselló y A. Femenia. Effect of air-drying temperature on physico-chemical properties of dietary fibre and antioxidant capacity of orange (Citrus aurantium v. Canoneta) by-products. Food Chemistry, 104(3), 1014-1024 (2007)

Jacho, A. y C. Vásquez. Efecto de una pectinasa e hidróxido de calcio en las características físico-químicas y sensoriales de jugo de sábila con naranja. Trabajo de grado (Ingeniería en agroindustria alimentaria), Universidad de Zamorano, Honduras (2011)

López-Córdoba, A., L. Deladino, L. Agudelo-Mesa y M. Martino. Yerba mate antioxidant powders obtained by co-crystallization: Stability during storage. Journal of Food Engineering, 124, 158-165 (2014)

López-Córdoba, A., L. Gallo, V. Bucala, M. Martino y A. Navarro. Co-crystallization of zinc sulfate with sucrose: A promissory strategy to render zinc solid dosage forms more palatable. Journal of Food Engineering 170, 100-107 (2016)

Manayay, D., A. Ibarz, W. Castillo, y L. Palacios. Cinética de la diferencia de color y croma en el proceso térmico de pulpa de mango (Mangifera indica L.) variedad Haden. Scientia Agropecuaria (4),181-190 (2013)

Martínez, J. y otros cuatro autores. Estabilidad de antocianinas en jugo y concentrado de agraz (Vaccinium meriodionale SW). Revista Facultad Nacional de Agronomía 64(1), 6-11 (2011)

Mendoza-Corvis, F., E. Hernandez, y L.E. Ruiz. Efecto del Escaldado sobre el Color y Cinética de Degradación Térmica de la Vitamina C de la Pulpa de Mango de Hilacha (Mangífera indica var magdalena river). Información tecnologica 26(3), 9-16 (2015)

Shimono, K., K. Kadota, Y. Tozuka, A. Shimosaka, Y. Shirakawa y J. Hidaka. Kinetics of co-crystal formation with caffeine and citric acid via liquid-assisted grinding analyzed using the distinct element method. European Journal of Pharmaceutical Sciences 76, 217-224 (2015)

Torregrosa, T., C. Cortés, M.J. Esteve, y A. Frígola. Effect of High-Intensity Pulsed Electric Fields Processing and Conventional Heat Treatment on Orange-Carrot Juice Carotenoids. Journal of Agricultural and Food Chemistry 53 (24), 9519-9525 (2005)

Varzakas, T. y T. Constantina. Handbook Food Processing: Food Safety, Quality and Manufacturing Processes. CRC Press (2015)

Yujing, S., S. Yan, L. Donghong y Y. Xingqian, Y. Effects of drying methods on phytochemical compounds and antioxidant activity of physiologically dropped un-matured citrus fruits. LWT - Food Science and Technology, 60(2), 1269-1275 (2014) 